\title{
Surtuin 1 as a potential prognostic biomarker in very elderly patients with colorectal cancer
}

\author{
Guk Jin Lee ${ }^{1}$, Yun Hwa Jung ${ }^{2}$, Tae-Jung Kim³, Yosep Chong ${ }^{3}$, Seo-Won Jeong ${ }^{4}$, In Kyu Lee ${ }^{5}$, and In Sook Woo ${ }^{6}$
}

\begin{abstract}
${ }^{1}$ Division of Medical Oncology, Department of Internal Medicine, Bucheon St. Mary's Hospital, College of Medicine, The Catholic University of Korea, Bucheon; ${ }^{2}$ Division of Medical Oncology, Department of Internal Medicine, Daejeon Sun Medical Center, Daejeon; ${ }^{3}$ Department of Hospital Pathology, ${ }^{4}$ Institute of Clinical Medical Research, Yeouido St. Mary's Hospital, College of Medicine, The Catholic University of Korea, Seoul; ${ }^{5}$ Department of Surgery, Seoul St. Mary's Hospital, College of Medicine, The Catholic University of Korea, Seoul; ${ }^{6}$ Division of Medical Oncology, Department of Internal Medicine, Yeouido St. Mary's Hospital, College of Medicine, The Catholic University of Korea, Seoul, Korea
\end{abstract}

Received: July 30, 2019

Revised : November 17, 2019

Accepted: July 1, 2020

\section{Correspondence to}

In Sook Woo, M.D.

Division of Medical Oncology,

Department of Internal

Medicine, Yeouido St. Mary's

Hospital, College of Medicine,

The Catholic University of Korea,

10 63-ro, Yeongdeungpo-gu,

Seoul 07345, Korea

Tel: +82-2-3779-1574

Fax: $+82-2-780-3132$

E-mail: insookwoo@catholic.ac.kr https://orcid.org/o000-0001-

$8220-2260$

Background/Aims: Colorectal cancer (CRC) rate increases with aging. Aging-related proteins, such as sirtuins (SIRTs) may be a potential therapeutic target in the elderly patients with CRC. The clinical implications of SIRT1 and SIRT2 have not been reported for elderly patients with cancer. The aim of this study was to evaluate the impact of expression of SIRT1 and SIRT2 on clinical outcome in two extreme age groups of patients with CRC.

Methods: The expression of SIRT1 and SIRT2 were evaluated in CRC tissues of 101 patients aged $\geq 80$ years and 29 patients aged $\leq 40$ years by immunohistochemistry. We defined the patients aged $\geq 80$ years as the very elderly and patients aged $\leq$ 40 years as the young patients. Correlations between the expression of these proteins and clinicopathological features were analyzed.

Results: The prognosis for the very elderly patients with high expressions of SIRT1 was significantly worse than that for patients showing low expression (median survival, 24.9 months vs. 38.6 months, $p=0.027$ ) whereas high expression of SIRT2 better prognosis (median survival, 37.9 months vs. 17.3 months, $p=0.006$ ). However, the young patients did not show any difference in prognosis according to expression of SIRT1 and SIRT2. In multivariate analysis, high SIRT1 expression retained statistical significance as a poor prognostic factor in the very elderly patients with CRC.

Conclusions: The results suggest that high SIRT1 expression could be predictive of a poor outcome for very elderly patients with CRC.

Keywords: Colorectal neoplasms; Sirtiun 1; Sirtuin 2; Aged, 8o and over

\section{INTRODUCTION}

The mammalian sirtuin (SIRT) family has homology with silent information regulator 2 (Sir2), which is a nicotinamide adenine dinucleotide (NAD+)-dependent deacetylase and extends the life span in yeast [1]. Human SIRT has seven isoforms (SIRT1-7) binding to various histone and non-histone proteins, and their function may differ according to substrates, acting as lysine deacetylase (SIRT1-3, 5, 6, 7), ADP ribosyl transferase (SIRT 4,6 ), and deacylase (SIRT5) $[2,3]$. SIRTs play an important role in normal cellular homeostasis through regulation of metabolism, mediation of autophagy, and maintenance of genetic stability [3,4]. SIRTs are also involved in age-associated diseases, such as metabolic syndrome, cardiovascular disease, neurodegeneration, and cancer [5]. The 
potential therapeutics targeting SIRT for the prevention and treatment of aging-associated cancers have been reported $[6,7]$.

Incidence of many cancers, including colon cancer increases with aging, and aging is a major risk factor in these cancers $[8,9]$. Approximately $24 \%$ of patients with colorectal cancer (CRC) are diagnosed at the age of 80 and older [10]. Aging-related proteins, such as SIRT, might have an important role in susceptibility to cancer in elderly patients. The role of SIRT1 and SIRT2 as tumor suppressors or promoters remains unclear in carcinogenesis and seems to vary according to the status of cell and pathologic process. SIRT1 among SIRT family has been studied the most widely for human cancer tissues, such as prostate cancer, breast cancer, non-smallcell lung cancer, leukemia, esophageal cancer and colon cancer [11-16]. SIRT1 may have dual roles through the activation of DNA repair: to keep genomic stability in normal cells and to help cancer cells survive and evolve to carcinogenesis with accumulation of mutations [3]. Recently, high expression of SIRT1 in colon cancer has been reported to be associated with vascular invasion and poor outcome in meta-analysis [16]. The median age of the patients with SIRT1 high expression and low expression was 63.1 to 64.8 years in the meta-analysis, respectively. SIRT2 also has been reported to have a dual role of protection and aggravation in cancer as follows. In glioma, SIRT2 has been suggested to play the role of tumor suppressor [17]. Also, SIRT2 knock-out mice developed genetic instability and hepatocellular carcinoma [18]. However, upregulation of SIRT2 was observed in primary acute leukemia blasts, and high expression of SIRT2 was associated with aggressiveness of prostate cancer $[19,20]$. In melanoma, SIRT2 was suggested to be involved in the process of metastasis in addition to cellular proliferation [21]. G1 arrest and destabilization of Snail was induced by SIRT2 in colon cancer cell lines and SIRT2 inhibition was suggested to be a potential therapeutic target for colon cancer [7]. The expression of SIRT1 and SIRT2 has not been investigated for a specific age group in patients with CRC so far.

We investigated for the first time the expression of SIRT1 and SIRT2 in the patients aged $\geq 80$ years and aged $\leq 40$ years with CRC to determine whether clinical significance differs according to the expression of these proteins in the extreme age group of CRC. The correla- tion between the two proteins of SIRT1 and SIRT2 was also evaluated in this study.

\section{METHODS}

\section{Patients and samples}

We performed a retrospective study of two cohorts (the very elderly vs. the young) of CRC patients with specimens and clinical records. Written informed consent was waived by the Institutional Review Board (IRB) because the patients were diagnosed with CRC between 1998 and 2012 before the revision of the Bioethics Act in February 2013. A total of 101 elderly ( $\geq 80$ years old) and 29 young ( $\leq 40$ years old) CRC patients were pathologically confirmed at the Yeouido St Mary's Hospital. Specimens were obtained from paraffin-embedded tissues stored in the Department of Pathology. Clinicopathological information of the two different age groups of patients was systematically reviewed and compared. Clinicopathologic data included stage, sex, performance status (PS), tumor location (right side vs. left side), histologic differentiation, comorbid diabetes mellitus (DM), and body mass index (BMI). The IRB of the Catholic Medical Center approved this study (IRB No. SC19SESIoo35).

\section{Tissue preparation and immunohistochemical staining}

The expression of SIRT1 and SIRT2 in tumor tissues was evaluated according to the following procedure. Specimens were obtained from filed paraffin-embedded tissues stored in the department of pathology. At first, SIRT1 and SIRT2 antigen retrieval were performed by heating under pressure in TE buffer ( 1 mM EDTA, 5 mM Tris, $\mathrm{pH}$ 8.o) for 5 minutes in a microwave. Immunohistochemical (IHC) staining was performed to determine the expression status of SIRT1 and SIRT2 protein using anti-SIRT1 and anti-SIRT2 rabbit monoclonal antibody (SIRT1: 1:100 dilution, SIRT2: 1:50 dilution, ab32441; Abcam Inc., Cambridge, MA, USA) according to the manufacturer's instructions. Slides were incubated with the respective primary antibodies in a moist chamber at room temperature overnight. In each run, a negative and a positive control were included. The signal was visualized using Envision (DAKO, Cambs, UK) 
and 3, 3'-diaminobenzididine (DAB, Vector Laboratories, Peterborough, UK). Sections were counterstained wit h hematoxylin.

\section{Evaluation of SIRT1 and SIRT2 expression}

Expression of SIRT1 and SIRT2 was evaluated using light microscopy by two independent pathologists. Discordant cases were reviewed on a multi-headed microscope to achieve consensus with confirmation of pathologist. Each slide was graded by the intensity (no staining: o point, weak staining: 1 point, moderate staining: 2 points, strong staining: 3 points) and the extent of staining (no staining of cells: $0,<25 \%$ of cells stained positive: $1,26 \%$ to $50 \%$ of cells stained positive: $2,51 \%$ to $75 \%$ of cells stained positive: $3,>75 \%$ of cells stained positive: 4). After that, the immune reactivity score (IRS) was calculated by multiplying two grades (intensity $x$ extent) according to the usual methods $[22,23]$. The final scores of tumors were categorized as negative or low expression (score $=0$ to 5 ) and high expression (score $\geq$ 6). The associations between clinicopathological factors and IRS of SIRT1 and SIRT2 were then investigated.

\section{Statistical analysis}

All statistical analyses were performed using the SPSS version 18.o for Windows (SPSS Inc., Chicago, IL, USA). Correlations between expression of SIRT proteins and the clinicopathological characteristics were analyzed by Pearson's chi-square tests. The Kaplan-Meier method with the log-rank test was used to calculate overall survival (OS) and compare it between high and low SIRT1/2 expression groups. OS was calculated as the time from the date of diagnosis of CRC to the date of death or last follow-up. Univariate and multivariate analyses for OS were performed using the Cox proportional hazard model to analyze independent prognostic factors. In all statistical analyses, $p<0.05$ was considered statistically significant.

\section{RESULTS}

\section{Patients characteristics}

The clinicopathological characteristics of all 130 (101 elderly patients, 29 young patients) patients are presented in Table 1. The median age of the elderly cohort and the young cohort were 82 years (range, 80 to 95 ) and 37 years (range, 28 to 40), respectively. The proportion of patients with DM was higher in the elderly than the young (29/101 [28.7\%] vs. 2/29 [6.9\%], $p=0.010)$. However, no significant differences were noted in other clinicopathological characteristics between two different age groups. The median follow-up period was 30.9 months (range, 0.5 to 76.9 ).

\section{Treatment}

In the very elderly patients, 61 (60.4\%) of 101 patients received surgery. Curative resection was performed for 52 (85.2\%), and palliative surgery was done for nine (14.8\%). Forty-seven (46.5\%) of the elderly patients received chemotherapy. Of these patients, 34 (72.3\%) patients received neoadjuvant or adjuvant chemotherapy and 13 (27.7\%) patients received palliative chemotherapy.

In the young patients, all 29 (100\%) patients received surgery. Curative resection was performed for 27 (93.1\%), and palliative surgery was done for two (6.9\%). Twenty-eight $(96.6 \%)$ of the young patients received chemotherapy. Neoadjuvant or adjuvant chemotherapy was prescribed for 25 (89.3\%) patients, and palliative chemotherapy was prescribed for three (10.7\%) patients.

\section{Association of SIRT1 and SIRT2 expression with clinicopathologic features of patients with CRC}

In tumor tissue, SIRT1 expression by IHC staining was mainly localized to the nucleus (Fig. 1A), and SIRT2 expression predominantly resided in the cytoplasm of cancer cells (Fig. 1B). Table 2 shows the association between SIRT1/2 expression status and clinicopathologic parameters of all 130 CRC patients. High expressions of SIRT1 and SIRT2 were seen in 58.5\% (76/130) and $51.6 \%$ (66/128, two missing) in all patients. When comparing the SIRT1 expression between two different age groups, the proportion of patients with high SIRT1 expression was significantly higher in the very elderly than in the young $(64.4 \%$ vs. $36.7 \%, p=0.011)$. Similarly, the proportion of patients with high SIRT2 expression was also significantly higher in elderly patients than in young patients $(57.4 \%$ vs. $26.7 \%, p=0.003)$. No statistically significant associations were identified between high SIRT1 expression and other clinicopathological features. However, high expression of SIRT2 was more commonly found in patients with DM compared with the non-diabetic patients $(67.7 \%$ vs. $45.5 \%, p=0.038)$. 
Table 1. Clinicopathologic characteristics of the very elderly and young colorectal cancer patients

\begin{tabular}{|c|c|c|c|}
\hline Characteristic & Elderly patients $(\mathrm{n}=101)$ & Young patients $(\mathrm{n}=29)$ & $p$ value \\
\hline Age, yr & $82(80-95)$ & $37(28-40)$ & \\
\hline Sex & & & $0.55^{8}$ \\
\hline Men & $53(52.5)$ & $17(58.6)$ & \\
\hline Women & $48(47 \cdot 5)$ & $12(41.4)$ & \\
\hline Body mass index, $\mathrm{kg} / \mathrm{m}^{2}$ & & & 0.723 \\
\hline$<20$ & $20(19.8)$ & $7(24.1)$ & \\
\hline $20-25$ & $48(47 \cdot 5)$ & $17(58.6)$ & \\
\hline$>25$ & $22(21.8)$ & $5(17.2)$ & \\
\hline Missing & $11(10.9)$ & & \\
\hline Tumor location & & & 0.065 \\
\hline Proximal (right, colon, T-colon) & $30(29.7)$ & $4(13.8)$ & \\
\hline Distal (left, colon, rectosigmoid) & $71(70.3)$ & $25(86.2)$ & \\
\hline Staging & & & 0.068 \\
\hline I & $18(17.8)$ & $3(10.3)$ & \\
\hline II & $27(26,7)$ & $14(48.3)$ & \\
\hline III & $23(22.8)$ & $8(27.6)$ & \\
\hline IV & $33(32.7)$ & $4(13.8)$ & \\
\hline Missing & $2(2.0)$ & & \\
\hline Histological differentiation & & & 0.119 \\
\hline Well or moderately & $91(91.1)$ & $23(79.3)$ & \\
\hline Poorly & $10(9.9)$ & $6(20.7)$ & \\
\hline Diabetes & & & $0.010^{\mathrm{a}}$ \\
\hline Present & $29(28.7)$ & $2(6.9)$ & \\
\hline Absent & $72(71.3)$ & $27(93.1)$ & \\
\hline
\end{tabular}

Values are presented as median (range) or number (\%).

T-colon, transverse colon.

${ }^{\mathrm{a}} \mathrm{p}<0.05$.
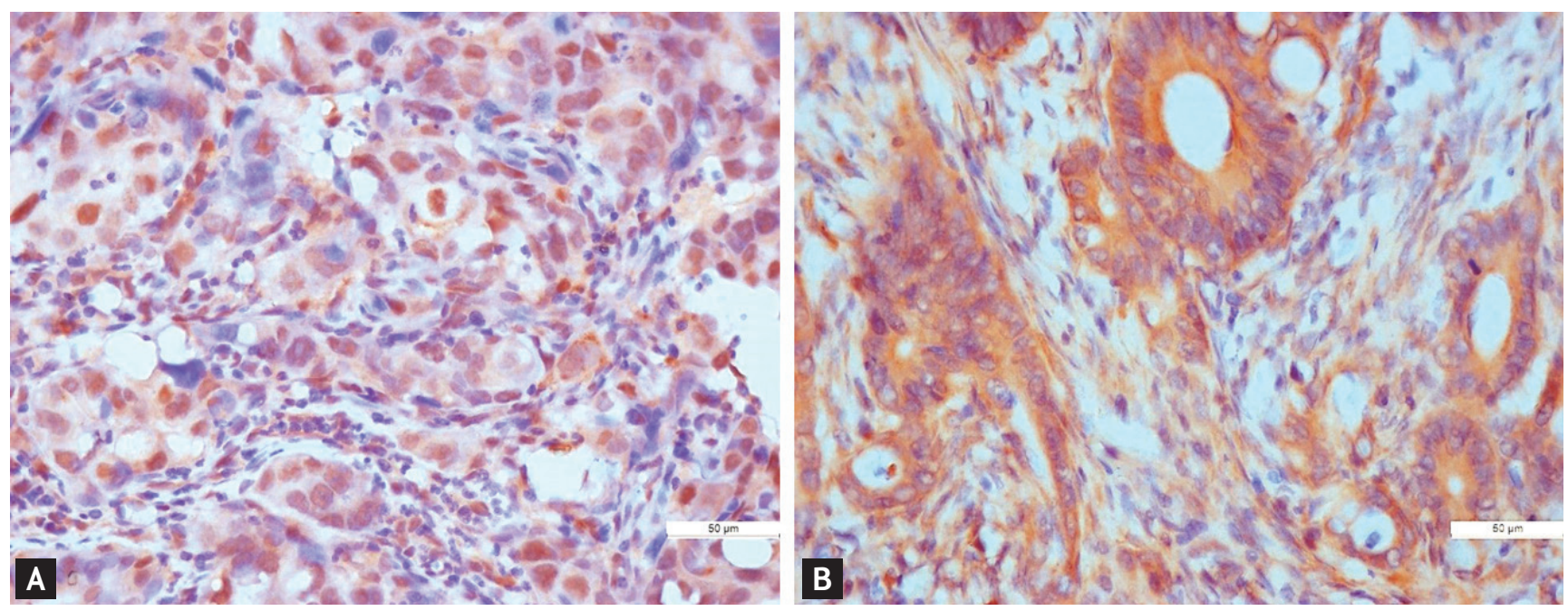

Figure 1. Immunohistochemical staining for sirtuin 1 (SIRT1) and sirtuin 2 (SIRT2) in colorectal cancer tissue ( $\times 400)$. (A) SIRT1: positive. (B) SIRT2: positive. 
Table 2. Correlation between the clinicopathologic parameters and SIRT1/2 expression in all patients

\begin{tabular}{|c|c|c|c|c|c|c|c|c|}
\hline \multirow{2}{*}{ Parameter } & \multirow{2}{*}{ Total } & \multicolumn{3}{|c|}{ SIRT1 } & \multicolumn{4}{|c|}{ SIRT2 } \\
\hline & & Low & High & $p$ value & Low & High & Missing & $p$ value \\
\hline All cases & 130 & 54 & 76 & & 62 & 66 & 2 & \\
\hline Age, yr & & & & $0.011^{\mathrm{a}}$ & & & & $0.003^{2}$ \\
\hline Elderly, $\geq 80$ & 101 & 36 & 65 & & 41 & 58 & 2 & \\
\hline Young, $<40$ & 29 & 18 & 11 & & 21 & 8 & & \\
\hline Sex & & & & 0.701 & & & & 0.706 \\
\hline Men & 70 & 28 & 42 & & 34 & 34 & 2 & \\
\hline Women & 60 & 26 & 34 & & 28 & 32 & & \\
\hline Body mass index, $\mathrm{kg} / \mathrm{m}^{2}$ & & & & 0.989 & & & & 0.194 \\
\hline$<20$ & 27 & 12 & 15 & & 12 & 8 & & \\
\hline $20-25$ & 65 & 28 & 37 & & 19 & 28 & & \\
\hline$>25$ & 27 & 12 & 15 & & 7 & 14 & & \\
\hline Missing & 11 & & & & & & & \\
\hline Tumor location & & & & 0.960 & & & & 0.540 \\
\hline Proximal (right, colon, T-colon) & 34 & 14 & 20 & & 18 & 16 & & \\
\hline Distal (left, colon, rectosigmoid) & 96 & 40 & 56 & & 44 & 50 & 2 & \\
\hline Staging & & & & 0.472 & & & & 0.664 \\
\hline I & 21 & 11 & 10 & & 9 & 12 & & \\
\hline II & 41 & 16 & 25 & & 17 & 23 & 1 & \\
\hline III & 31 & 10 & 21 & & 16 & 14 & 1 & \\
\hline IV & 37 & 17 & 20 & & 20 & 17 & & \\
\hline Histological differentiation & & & & 0.726 & & & & 0.229 \\
\hline Well or moderately & 114 & 48 & 66 & & 52 & 60 & 2 & \\
\hline Poorly & 16 & 6 & 10 & & 10 & 6 & & \\
\hline Diabetes & & & & 0.230 & & & & $0.038^{\mathrm{a}}$ \\
\hline Present & 31 & 10 & 21 & & 10 & 21 & & \\
\hline Absent & 99 & 44 & 55 & & 52 & 45 & 2 & \\
\hline
\end{tabular}

Values are presented as number.

SIRT1, sirtuin 1; SIRT2, sirtuin 2; T-colon, transverse colon. ${ }^{\mathrm{a}} p<0.05$.

\section{Prognostic significance of expression of SIRT1 and SIRT2 in CRC patients}

For all 130 patients with CRC, high expression of SIRT1 was associated with shorter OS in comparison using the log-rank test. However, the expression of SIRT2 was not associated with prognosis. In the very elderly patients with CRC, high expression of SIRT1 was consistently associated with shorter OS (median OS, 24.9 months vs. 38.6 months, $p=0.027$ ), but high expression of SIRT2 was associated with longer OS (median OS, 37.9 months vs. 17.3 months, $p=0.006$ ) (Fig. 2). In the young patients, high expressions of SIRT1 and SIRT2 were not associated with OS (median OS, 76.9 months vs. 61 months, $p=0.250$; median OS, 56.4 months vs. 61.4 months, $p=$ 0.658). Expression of SIRT1 and SIRT2 were associated with prognosis in the very elderly, not the young patients, and the young patients tended to be opposite to the elderly patients. In univariate survival analysis, BMI (> 25), good PS (O to 1), localized disease (stage I to III), and well-differentiated histologic grade, low SIRT1 expression, and high SIRT2 expression were significantly correlated with longer OS (Table 3). In multivariate sur- 

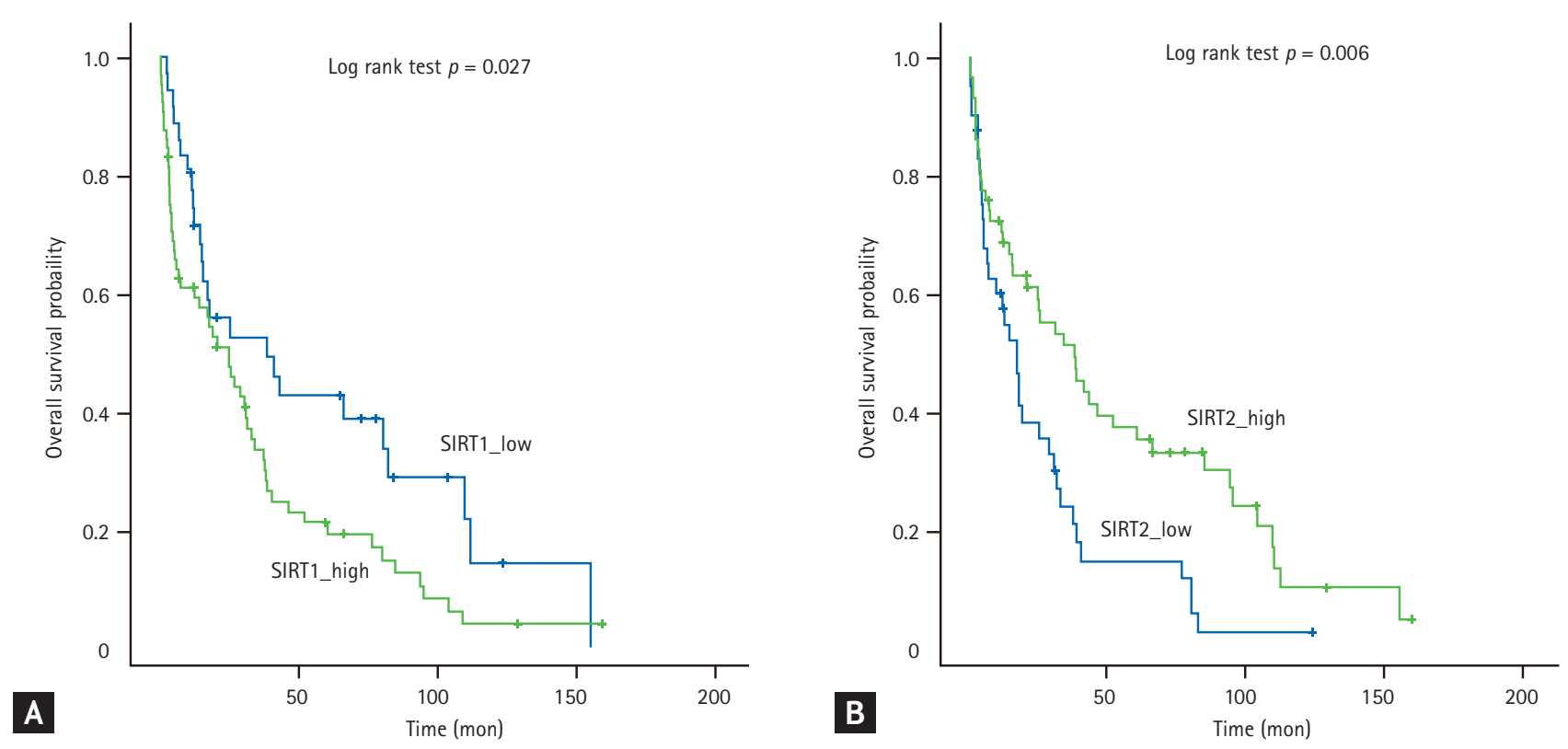

Figure 2. Factors affecting the overall survival of the very elderly patients with colorectal cancer. (A) Sirtuin 1 (SIRT1) expression. (B) Sirtuin 2 (SIRT2) expression.

Table 3. Univariate analysis of variables affecting the overall survival in the very elderly patients with colorectal cancer $(\mathrm{n}=101)$

\begin{tabular}{lccc}
\hline \multirow{2}{*}{ Variable } & No. of patients & Overall survival & \multicolumn{2}{c}{$p$ value } \\
\cline { 3 - 4 } Male & 53 & $0.908(0.583-1.415)$ & 0.670 \\
BMI $>25 \mathrm{~kg} / \mathrm{m}^{2}$ & 22 & $0.523(0.290-0.942)$ & $0.031^{\mathrm{a}}$ \\
PS o-1 & 62 & $0.207(0.126-0.339)$ & $0.000^{\mathrm{a}}$ \\
Location: proximal & 30 & $1.145(0.708-1.850)$ & 0.581 \\
Stage: localized & 68 & $0.186(0.113-0.309)$ & $0.000^{\mathrm{a}}$ \\
WD/MD & 91 & $0.285(0.144-0.567)$ & $0.000^{\mathrm{a}}$ \\
DM & 29 & $0.701(0.426-1.154)$ & 0.701 \\
SIRT1 high & 65 & $1.699(1.057-2.733)$ & $0.029^{\mathrm{a}}$ \\
SIRT2 high & 58 & $0.534(0.338-0.841)$ & $0.007^{\mathrm{a}}$ \\
\hline
\end{tabular}

HR, hazard ratio; CI, confidence interval; BMI, body mass index; PS, performance status; WD, well-differentiated; MD, moderately differentiated; PD, poorly differentiated; DM, diabetes mellitus; SIRT1, sirtuin 1; SIRT2, sirtuin 2.

$\mathrm{a} p<0.05$.

vival analysis, BMI (> 25), good PS (o to 1), and localized disease (stage I to III) were associated with longer OS, while SIRT1 overexpression was associated with shorter OS (95\% confidence interval, 1.101 to $3.129 ; p=0.020$ ) (Table 4).

\section{DISCUSSION}

Association between carcinogenesis and the biology of aging is still under investigation. Accumulation of mutations in cells and changes of the tissue microenvironment with aging may cause cells to develop into malignancies, whereas aging of vascular and immune system or changes of growth and sex hormones may suppress 
Table 4. Multivariate analysis of variables affecting the overall survival in the very elderly patients with colorectal cancer $(n=101)$

\begin{tabular}{lcc}
\hline \multirow{2}{*}{ Variable } & \multicolumn{2}{c}{ Overall survival } \\
\cline { 2 - 3 } & HR $(95 \%$ CI $)$ & $p$ value \\
PS $>25 \mathrm{~kg} / \mathrm{m}^{2}$ & $0.433(0.232-0.807)$ & 0.008 \\
Localized stage & $0.269(0.153-0.472)$ & 0.000 \\
WD/MD & $0.183(0.097-0.347)$ & 0.000 \\
SIRT1 high & $0.305(0.291-1.471)$ & 0.305 \\
SIRT2 high & $1.856(1.101-3.129)$ & 0.020 \\
\hline
\end{tabular}

HR, hazard ratio; CI, confidence interval; BMI, body mass index; PS, performance status; WD, well-differentiated; MD, moderately differentiated; PD, poorly differentiated; SIRT1, sirtuin 1; SIRT2, sirtuin 2.

cancer progression [8]. Because increasing age is a significant risk factor of many cancers, SIRTs implicated in longevity might play a crucial role in carcinogenesis of aging-associated cancers [24]. Subcellular location of SIRT varies according to the isoform of SIRT, SIRT1 and SIRT2 are predominantly located in nucleus and cytoplasm, respectively [3].

We analyzed the impact of expression of SIRT1 and SIRT2 on outcome in the very elderly patients with CRC. In this study, SIRT1 and SIRT2 overexpression were more commonly observed in tumor tissues of the very elderly CRC patients than young patients. To our knowledge, the expression of SIRT for specific age groups of CRC patients have never been reported, although one study analyzed the change of SIRT expression status in colon tissue along with the aging process in rats [25]. At present, it is unclear whether the aging enhanced expression of SIRT1 or malignant transformation can be attributed to the high expression of SIRT1 in the colon cancer tissue of elderly patients. It was proposed that hypermethylated in cancer 1 ( $\mathrm{HIC1}$ ) repress transcription of $\mathrm{SIRT} 1$, and $\mathrm{HICl}_{1}$ promoter is epigenetically silenced by hypermethylation along with aging $[26,27]$. Consequently, decrement of $\mathrm{HIC1}$ expression with aging can result in an upregulated SIRTi level in elderly persons, and increased SIRT1 expression may contribute to age-related carcinogenesis in CRC. Specifically, SIRT1 is reported to deacetylate a few apoptosis-related transcription factors, such as tumor protein p53 (p53) and mammalian forkhead box O (FOXO), to balance repair and apoptosis of cells $[28,29]$. Thus, inactivation of p53-mediated apoptosis by SIRT1 may promote cell survival after oxidative damage, leading to the accumulation of mutations and carcinogenesis [30]. This hypothesis supports the results that the proportion of patients with high SIRT1 expression was higher in the very elderly CRC patients in our study and suggests that SIRT1 protein may play a more important role in the carcinogenesis of elderly CRC patients than that in young patients. Nevertheless, many previous clinical studies did not show a significant difference of SIRT1 expression in CRC patients according to age [31]. In those studies CRC patients were usually categorized into two age groups at the age of 60 or 65. However, we compared the very elderly CRC patients aged $\geq 80$ years with the yonger patients, aged $\leq$ 40 years to assess the impact of age on the expression of age-related proteins, SIRT1 and SIRT2. Nosho et al. [32] reported that SIRT1 expression is associated with CpG island methylator phenotype (CIMP) and microsatellite instability (MSI)-high subtype in CRC patients. The study included mostly elderly patients aged over 60 years old. Both CIMP-high and MSI-high phenotype are more commonly identified in older CRC patients, which is consistent with our results. Our study showed that SIRT2 expression was also higher in the very elderly patients than in the young patients. Increased expression of SIRT2 was reported to be a marker of cellular senescence in a stress-induced premature senescence model [33]. Increased expression of SIRT2 in malignant tissue of elderly CRC patients also may be a consequence of senescence.

Activation of SIRT1 deacetylates target proteins involved in gluconeogenesis and regulates diabetes by increasing insulin secretion from pancreatic $\beta$-cell and insulin sensitivity. Therefore, SIRT1 has been suggested as a potential target for treating diabetes [4]. SIRT2 in- 
teracts with the AKT pathway, which is important for insulin-resistant diabetes as well as carcinogenesis. SIRT2 inhibition blocks AKT activation; however, SIRT2 overexpression sensitizes cells to insulin. The study suggests that SIRT2 could also be a useful target in the treatment of type 2 diabetes [34]. Association between high SIRT2 expression and DM was observed in our study. However, the exact mechanism by which the patients with high expression of SIRT2 showed higher prevalence of diabetes remains unclear.

Our study revealed that high SIRT1 expression is associated with worse prognosis in the very elderly patients with CRC. The finding suggests that age might affect the expression of SIRT1 in CRC. SIRT1 is known to be an anti-apoptotic protein and is usually overexpressed in several cancers, including CRC. Indeed, a few studies have reported that SIRT1 overexpression is related to poor outcome or disease progression in CRC patients [14]. By contrast, the others showed that SIRT1 overexpression is also related to better outcome in some CRC patients $[31,35,36]$. Although at present the exact role of SIRT1 as a prognostic biomarker has not been established in CRC patients, based on a recent meta-analysis, SIRT1 as a negative prognostic factor in CRC seems get the upper hand, and another meta-analysis suggested that SIRT1 acts as a tumor promoter in CRC [37]. We analyzed the prognostic significance of SIRT1 and SIRT2 in two extremely different age groups to evaluate the impact of age on the expression of these proteins. High SIRT2 expression was associated with better prognosis in elderly CRC patients. SIRT2 has been linked with mitosis because it deacetylases microtubules $(\alpha$-tubulin) [38]. SIRT2 is located primarily in the cytoplasm, but occasional migration of SIRT2 into the nucleus is identified during cancerous transformation or exposure to ionizing radiation [39]. The loss of SIRT2 is expected to promote genomic instability as an early event in carcinogenesis. In addition to SIRT2's role as a tumor suppressor, the role of SIRT2 as a tumor promotor in carcinogenesis has been reported [40,41]. Stabilization of myc oncoproteins by SIRT2 protein has been reported in carcinogenesis [42]. Because SIRT1 and SIRT2 share substrates, such as $\mathrm{p}_{53}$, FOXO, or myc, there might be interaction between SIRT1 and 2 [7,43-45]. Therefore, we investigated to find associations between SIRT1 and SIRT2 in the CRC patients in this study, but no associa- tion was found.

In conclusion, our study shows that overexpression of SIRT1 and SIRT2 was observed more commonly in the very elderly patients than in young patients with CRC. Age may affect the expression of SIRT proteins in two extreme age groups of patients with CRC. SIRT1 overexpression was associated with poor outcome in the very elderly patients with CRC. The results suggest for the first time that high SIRT1 expression may be predictive of a poor outcome and SIRT1 could become a potential therapeutic target for the very elderly patients with CRC in the future.

\section{KEY MESSAGE}

1. Sirtuin 1 (SIRT 1) and SIRT2 are more overexpressed in the very elderly patients with colorectal cancer (CRC).

2. High expressions of SIRT1 suggests poor prognosis in the very elderly patients with CRC.

3. SIRT1 could become a potential therapeutic target for the very elderly patients with CRC.

\section{Conflict of interest}

No potential conflict of interest relevant to this article was reported.

\section{Acknowledgments}

This research was supported by a grant from the Institute of Clinical Medicine Research in the Yeouido St. Mary's Hospital, Catholic University of Korea (Grant No. YSI2015-06).

\section{REFERENCES}

1. Guarente L, Kenyon C. Genetic pathways that regulate ageing in model organisms. Nature 2000;408:255-262.

2. Bosch-Presegue L, Vaquero A. The dual role of sirtuins in cancer. Genes Cancer 2011;2:648-662.

3. Roth M, Chen WY. Sorting out functions of sirtuins in cancer. Oncogene 2014;33:1609-1620.

4. Chalkiadaki A, Guarente L. Sirtuins mediate mammalian metabolic responses to nutrient availability. Nat Rev Endocrinol 2012;8:287-296. 
5. Hall JA, Dominy JE, Lee Y, Puigserver P. The sirtuin family's role in aging and age-associated pathologies. J Clin Invest 2013;123:973-979.

6. Liu T, Liu PY, Marshall GM. The critical role of the class III histone deacetylase SIRT1 in cancer. Cancer Res 2009;69:1702-1705.

7. Cheon MG, Kim W, Choi M, Kim JE. AK-1, a specific SIRT2 inhibitor, induces cell cycle arrest by downregulating Snail in HCT116 human colon carcinoma cells. Cancer Lett 2015;356(2 Pt B):637-645.

8. de Magalhaes JP. How ageing processes influence cancer. Nat Rev Cancer 2013;13:357-365.

9. Jung KW, Won YJ, Kong HJ, Lee ES. Cancer statistics in Korea: incidence, mortality, survival, and prevalence in 2016. Cancer Res Treat 2019;51:417-430.

10. Siegel R, Desantis C, Jemal A. Colorectal cancer statistics, 2014. CA Cancer J Clin 2014;64:104-117.

11. Huffman DM, Grizzle WE, Bamman MM, et al. SIRT1 is significantly elevated in mouse and human prostate cancer. Cancer Res 2007;67:6612-6618.

12. Derr RS, van Hoesel AQ, Benard A, et al. High nuclear expression levels of histone-modifying enzymes LSD1, HDAC2 and SIRT1 in tumor cells correlate with decreased survival and increased relapse in breast cancer patients. BMC Cancer 2014;14:604.

13. Han L, Liang XH, Chen LX, Bao SM, Yan ZQ. SIRT1 is highly expressed in brain metastasis tissues of non-small cell lung cancer (NSCLC) and in positive regulation of NSCLC cell migration. Int J Clin Exp Pathol 2013;6:23572365 .

14. Chen W, Bhatia R. Roles of SIRT1 in leukemogenesis. Curr Opin Hematol 2013;20:308-313.

15. Ma MC, Chiu TJ, Lu HI, et al. SIRT1 overexpression is an independent prognosticator for patients with esophageal squamous cell carcinoma. J Cardiothorac Surg 2018;13:25.

16. Hong WG, Pyo JS. The clinicopathological significance of SIRT1 expression in colon cancer: an immunohistochemical study and meta-analysis. Pathol Res Pract 2018;214:1550-1555.

17. Hiratsuka M, Inoue T, Toda T, et al. Proteomics-based identification of differentially expressed genes in human gliomas: down-regulation of SIRT2 gene. Biochem Biophys Res Commun 2003;309:558-566.

18. Kim HS, Vassilopoulos A, Wang RH, et al. SIRT2 maintains genome integrity and suppresses tumorigenesis through regulating APC/C activity. Cancer Cell
2011;20:487-499.

19. Dan L, Klimenkova O, Klimiankou M, et al. The role of sirtuin 2 activation by nicotinamide phosphoribosyltransferase in the aberrant proliferation and survival of myeloid leukemia cells. Haematologica 2012;97:551-559.

20. Hou H, Chen W, Zhao L, et al. Cortactin is associated with tumour progression and poor prognosis in prostate cancer and SIRT2 other than HADC6 may work as facilitator in situ. J Clin Pathol 2012;65:1088-1096.

21. Wilking-Busch MJ, Ndiaye MA, Huang W, Ahmad N. Expression profile of SIRT2 in human melanoma and implications for sirtuin-based chemotherapy. Cell Cycle 2017;16:574-577.

22. Remmele W, Stegner HE. Recommendation for uniform definition of an immunoreactive score (IRS) for immunohistochemical estrogen receptor detection (ER-ICA) in breast cancer tissue. Pathologe 1987;8:138-140.

23. Meyerholz DK, Beck AP. Principles and approaches for reproducible scoring of tissue stains in research. Lab Invest 2018;98:844-855.

24. Wu X, Cao N, Fenech M, Wang X. Role of sirtuins in maintenance of genomic stability: relevance to cancer and healthy aging. DNA Cell Biol 2016;35:542-575.

25. Akbulut KG, Aktas SH, Akbulut H. The role of melatonin, sirtuin2 and FoXO1 transcription factor in the aging process of colon in male rats. Biogerontology 2015;16:99-108.

26. Chen WY, Wang DH, Yen RC, Luo J, Gu W, Baylin SB. Tumor suppressor HIC1 directly regulates SIRT1 to modulate p53-dependent DNA-damage responses. Cell 2005;123:437-448.

27. Lin Z, Fang D. The roles of SIRT1 in cancer. Genes Cancer 2013;4:97-104.

28. Lee JT, Gu W. SIRT1: regulator of p53 deacetylation. Genes Cancer 2013;4:112-117.

29. Vaziri H, Dessain SK, Ng Eaton E, et al. hSIR2(SIRT1) functions as an NAD-dependent p53 deacetylase. Cell 2001;107:149-159.

30. Yi J, Luo J. SIRT1 and p53, effect on cancer, senescence and beyond. Biochim Biophys Acta 2010;1804:1684-1689.

31. Jung W, Hong KD, Jung WY, et al. SIRT1 expression is associated with good prognosis in colorectal cancer. Korean J Pathol 2013;47:332-339.

32. Nosho K, Shima K, Irahara N, et al. SIRT1 histone deacetylase expression is associated with microsatellite instability and $\mathrm{CpG}$ island methylator phenotype in colorectal cancer. Mod Pathol 2009;22:922-932. 
33. Anwar T, Khosla S, Ramakrishna G. Increased expression of SIRT2 is a novel marker of cellular senescence and is dependent on wild type p53 status. Cell Cycle 2016;15:18831897.

34. Ramakrishnan G, Davaakhuu G, Kaplun L, et al. Sirt2 deacetylase is a novel AKT binding partner critical for AKT activation by insulin. J Biol Chem 2014;289:60546066.

35. Firestein R, Blander G, Michan S, et al. The SIRT1 deacetylase suppresses intestinal tumorigenesis and colon cancer growth. PLoS One 2008;3:e2020.

36. Jang SH, Min KW, Paik SS, Jang KS. Loss of SIRT1 histone deacetylase expression associates with tumour progression in colorectal adenocarcinoma. J Clin Pathol 2012;65:735-739.

37. Zu G, Ji A, Zhou T, Che N. Clinicopathological significance of SIRT1 expression in colorectal cancer: a systematic review and meta analysis. Int J Surg 2016;26:32-37.

38. Dryden SC, Nahhas FA, Nowak JE, Goustin AS, Tainsky MA. Role for human SIRT2 NAD-dependent deacetylase activity in control of mitotic exit in the cell cycle. Mol Cell Biol 2003;23:3173-3185.
39. North BJ, Marshall BL, Borra MT, Denu JM, Verdin E. The human Sir2 ortholog, SIRT2, is an NAD+-dependent tubulin deacetylase. Mol Cell 2003;11:437-444.

40. McGlynn LM, Zino S, MacDonald AI, et al. SIRT2: tumour suppressor or tumour promoter in operable breast cancer? Eur J Cancer 2014;50:290-301.

41. Seo KS, Park JH, Heo JY, et al. SIRT2 regulates tumour hypoxia response by promoting HIF-1 $\alpha$ hydroxylation. Oncogene 2015;34:1354-1362.

42. Liu PY, Xu N, Malyukova A, et al. The histone deacetylase SIRT2 stabilizes Myc oncoproteins. Cell Death Differ 2013;20:503-514.

43. Yang MH, Laurent G, Bause AS, et al. HDAC6 and SIRT2 regulate the acetylation state and oncogenic activity of mutant K-RAS. Mol Cancer Res 2013;11:1072-1077.

44. Nguyen P, Lee S, Lorang-Leins D, Trepel J, Smart DK. SIRT2 interacts with $\beta$-catenin to inhibit Wnt signaling output in response to radiation-induced stress. Mol Cancer Res 2014;12:1244-1253.

45. Peck B, Chen CY, Ho KK, et al. SIRT inhibitors induce cell death and p53 acetylation through targeting both SIRT1 and SIRT2. Mol Cancer Ther 2010;9:844-855. 